\title{
¿Cuánto rentan realmente las AFP en Chile?
}

\author{
Claudio A. Bonilla ${ }^{a}$ y Carlos P. Maquieira ${ }^{b}$ \\ a Universidad de Chile, Chile \\ ${ }^{b}$ Altainver Consultores, Chile
}

\begin{abstract}
RESUMEN: En el presente artículo explicamos por qué el comparar la rentabilidad contable de las AFP con su tasa de costo de capital — como lo hace López (2016) — no es una comparación válida y en consecuencia no permite concluir que en dicha industria haya excesos de rentabilidad. En nuestro estudio ampliamos el ejercicio de López — basado solo en una AFP - a 29 empresas de distintos sectores y demostramos que el diferencial CAPM-ROE puede ser positivo o negativo, pero en ningún caso las AFP muestran un diferencial distinto al de otras industrias que operan en Chile. Es clave tener presente la importancia de dicho ejercicio desde la perspectiva de las políticas públicas, pues cualquier regulación futura en dicha industria debiera tener despejado este punto. Creemos que los resultados del presente ejercicio, realizado con la metodología estándar para el cálculo de la tasa de costo de capital y con los datos bursátiles de las compañías hasta diciembre de 2019, cierran el debate respecto de la inexistente rentabilidad sobrenormal en la industria de las AFP. Creemos que la forma correcta de ocuparse del tema de las pensiones debe ser a través de políticas
\end{abstract}

Claudio Bonilla es PhD en Economía de la University of Texas at Austin, Estados Unidos, e ingeniero en Información y Control de Gestión de la Universidad de Chile. Es Profesor Titular de la Facultad de Economía y Negocios, Universidad de Chile, y socio de Bonilla y Asociados Consultores. Dirección: Diagonal Paraguay 265, of. 1906, CP 8330015. Email: cbonilla@fen. uchile.cl.

Carlos Maquieira es PhD en Administración (Finanzas) de la University of Georgia, Estados Unidos, e ingeniero comercial de la Universidad de Chile. Es socio fundador de Altainver Consultores. Es Profesor Jornada Completa de CENTRUM Graduate School of Business, PUCP (Lima, Perú). Es además Profesor Asociado de la Universidad de Chile, donde ocupó diversos cargos directivos, entre los cuales destaca el de director de la Escuela de Posgrado de la Facultad de Economía y Negocios de la misma universidad. Dirección: Los Alerces 7231, Peñalolén, Santiago, Chile, CP 7910000. Email: cmaquieira@mqa.cl. 
públicas que incentiven un mayor ahorro por parte de los trabajadores y empleadores, y mediante el fortalecimiento del pilar solidario. Una regulación sobre el nivel de rentabilidad de una industria que no tiene características de monopolio natural, sino más bien de industria competitiva, sería un absurdo si no se sustenta en la teoría económica. PalABRAS CLAVE: AFP, rentabilidad, CAPM, competencia, políticas públicas RECIBIDO: septiembre 2020 / ACEPTADO: junio 2021

\section{How much Is the Real Economic Profitability of Pension Fund Administrators (AFPs) in Chile?}

ABSTRACT: In this article we explain why comparing the accounting profitability of the AFP with their cost of capital-as López (2016) does-is not a valid comparison and consequently does not allow us to conclude that there are excess profitability in this industry. In our study we extend López's exercise-based only on one AFP_to 29 companies from different sectors and show that the CAPM-ROE differential can be positive or negative, but in no case the AFP show a differential different from that of other industries that operate in Chile. The importance of this exercise is essential from the perspective of public policies, since any future regulation in that industry should have this point cleared. We believe that the results of this exercise, carried out with the standard methodology for calculating the cost of capital and with the stock market data of the companies until December 2019, close the debate regarding the non-existent excess profitability in the AFP industry. We believe that the correct way to think about future pensions is through public policies that encourage higher savings rates by workers and employers and through strengthening the solidarity pillar. A regulation center on the level of profitability of an industry that does not have the characteristics of a natural monopoly, but rather a competitive industry, would be an absurdity without support in economic theory.

KEYWORDs: pension funds, profitability, CAPM, competition, public policy

ReCeIved: September 2020 / AcCEPTED: June 2021

o hay duda de que uno de los problemas que aqueja a los países,
cualquiera sea su nivel de desarrollo, es el de las pensiones. ¿Qué régimen de pensiones desarrollar? ¿Cómo financiar buenas pensiones? ¿Cuál es el impacto en las cuentas fiscales ante cambios en el sistema de pensiones? ¿Cómo avanzar en el mix apropiado de financiamiento de pensiones entre el ahorro individual y el financiamiento público? Estas preguntas vuelven cada cierto tiempo, dado que no existe $-y$ probablemente no existirá nunca - 'la receta perfecta' para tratar el tema del 
envejecimiento de la población, su evolución demográfica y el financiamiento de las pensiones.

Nuestro país, después de treinta años de un avance histórico en su desarrollo económico, que nos hizo progresar desde un PIB per cápita (PPP corriente) de USD 4.500 el año 1990 hasta los USD 25.974 al año 2019, según datos del Banco Mundial, ${ }^{1}$ no está ajeno al debate sobre las pensiones, en particular ahora, que el nivel de desarrollo alcanzado nos permite ocuparnos de temas que, cuando éramos un país pobre, estaban fuera del alcance de la billetera fiscal debido a las necesidades aún más básicas y prioritarias de gasto público.

En tiempos en que se discuten importantes reformas a los sistemas de pensiones en Latinoamérica y en Chile en particular, nos parece adecuado desmitificar ciertas ideas que pueden terminar por desvirtuar la realidad del sistema de pensiones chileno, por ser este la referencia obligada para el resto de los países de la región. En particular, queremos analizar objetivamente la rentabilidad de las Administradoras de Fondos de Pensiones (AFP en adelante) para saber si efectivamente en esta industria las rentabilidades son excesivas respecto de lo que deberían ser. Para realizar esta comparación, vamos a tomar como punto de partida la metodología del López (2016) y la vamos a aplicar a varias empresas de distintas industrias en el país para comparar la rentabilidad sobre el patrimonio con el costo patrimonial estimado vía el modelo CAPM (Capital Asset Pricing Model), tal cual lo hace López (2016) para llegar a sus conclusiones. Adicionalmente, en el Anexo técnico incluimos, utilizando la correcta metodología, el desarrollo necesario para llegar desde el ROE (ratio de retorno sobre el patrimonio) a la rentabilidad económica realizada, que será comparada con la rentabilidad exigida del patrimonio.

López (2016) realiza un análisis de las rentabilidades de las AFP desde dos dimensiones. Por un lado, calcula el ROE para el período 2006 a 2015 para el sistema de AFP; posteriormente calcula la tasa exigida por los accionistas de las AFP para el mismo período usando el modelo CAPM, ampliamente usado en valoración de empresas y en determinación de tarifas en mercados regulados (monopolios naturales) como el mercado energético, de telecomunicaciones o el sanitario, por ejemplo. La principal conclusión de dicho estudio es que para el período analizado, los accionistas de las AFP han ganado 4,8 veces más (al mirar el ROE)

1 Información disponible en: https://data.worldbank.org/indicator/NY.GDP.PCAP. PP.CD?locations=CL [12 de octubre 2021]. 
de lo que deberían haber ganado según su exposición al riesgo del mercado (mirando el costo patrimonial), lo cual se deriva de la aplicación del modelo CAPM.

En el presente estudio argumentamos que el ROE no es comparable con la tasa exigida vía el modelo CAPM. El ROE se calcula con información histórica del desempeño contable de una compañía, mientras que el CAPM tiene como base la información económica de retornos tanto para la firma como para el mercado. Por otro lado, el CAPM tiene una visión futura respecto de la tasa exigida mínima, que los inversionistas requieren de una inversión dado su nivel de riesgo. Para demostrar lo anterior, en este artículo seguimos la metodología de López (2016) y comparamos ROE con la tasa exigida del patrimonio obtenida del modelo CAPM para distintas empresas en distintas industrias. Cuando se compara ROE con el costo patrimonial obtenido a través de CAPM las diferencias que se observan son positivas y negativas, dependiendo de la empresa que se considere, con resultados muy diversos. Además, observamos que la industria de las AFP está lejos de representar el diferencial más grande entre ROE y tasa exigida. Otras industrias como la bancaria o incluso empresas de sectores regulados (monopolios naturales cuyas tarifas son fijadas por ley), tienen un mayor diferencial entre el ROE y sus tasas exigidas, y por lo tanto, la idea de excesos de rentabilidad esgrimida a partir de la brecha entre ambos conceptos de rentabilidad no es económicamente correcta y en consecuencia no debiera considerarse como una muestra de rentabilidades excesivas o como una falta de competencia a la hora de legislar una nueva regulación para la industria de las AFP. Con esto no queremos decir que no haya que promover la competencia; todo lo contrario: más competencia es siempre bienvenida en cualquier industria, en particular en la industria de las AFP. Lo que sostenemos es que la comparación realizada por López (2016) no es una comparación válida, como veremos más adelante, y en consecuencia no debiera ser considerara por el regulador o como insumo a nivel parlamentario.

Desde nuestra perspectiva, cualquier cambio aplicado a la industria de AFP debiera propender a mejorar las pensiones y a profundizar la competencia en dicho mercado. Excesos de retornos como los calculados por López (2016) no debieran ser una variable a considerar, de acuerdo a lo que exponen los múltiples casos que analiza el presente artículo. La comparación entre ROE y tasas de retornos exigidas mínimas por los dueños del patrimonio, estimadas a través del CAPM, no es una comparación adecuada. 


\section{Evolución de la industria y comisiones}

A principios de la década de 1980 existían doce AFP en Chile. Muchas de ellas se fusionaron en los años siguientes, llegando a mantenerse cinco AFP hacia el año 2010. Ese mismo año, ocurre la primera licitación de afiliados nuevos del sistema, con lo cual se incorpora AFP Modelo a la industria, y se produce una importante baja general de las comisiones promedio cobradas por las AFP producto de la licitación. El proceso de licitación de la cartera nueva de las AFP se produce después de varios años de discusión respecto de cómo aumentar la competencia en la industria de las AFP. Esta discusión estaba basada en el principio de que existía una baja competencia en las comisiones de las AFP y en cierta insensibilidad hacia las comisiones de parte de los afiliados (Marcel et al. 2008). Actualmente existen siete AFP: Capital, Cuprum, Habitat, Modelo, Planvital, Provida y la nueva AFP Uno.

No sabemos cuál es el número óptimo de AFP para una industria como la chilena, pero lo que sí sabemos es que ha existido una evolución hacia la baja en la comisión promedio cobrada por las AFP, en particular después de la licitación de afiliados nuevos como se observa en el Gráfico 1.

Gráfico 1. EVOLUCIÓN DE COMISIONES

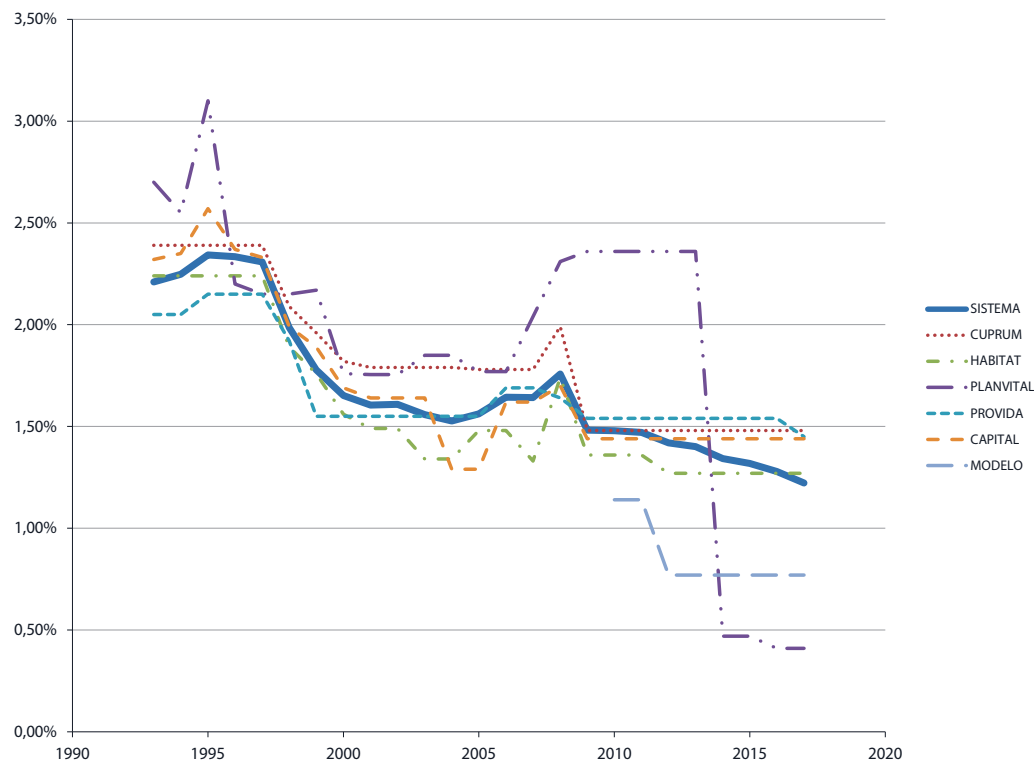

Fuente: Elaboración propia en base a datos de la Superintendencia de Pensiones de Chile. 
En el Gráfico 1 observamos cómo las comisiones cobradas por las AFP han bajado más de un $45 \%$ en los últimos veinte años, lo cual es un indicador indirecto de que ha existido un grado de competencia entre ellas, no solo en cuanto a la rentabilidad de sus fondos y respecto de la calidad del servicio que entregan, sino también en relación al impulso de un cobro menor de comisiones fundamentalmente a causa de las licitaciones de los afiliados. Del mismo modo, sabemos que no son las menores comisiones ni el mejor servicio las variables que impactan en el nivel de las pensiones futuras, pero de todas formas la baja en dichas comisiones es indicio de un mercado en el cual sus participantes compiten, hasta cierto punto, en el factor precio.

\section{El modelo CAPM y su uso en el mercado de capitales de Chile}

El modelo CAPM fue desarrollado por Sharpe (1964) y Lintner (1965) en un contexto de mercado de capitales perfecto y en un mundo de dos períodos. El modelo se basa en la teoría de portafolio media-varianza desarrollada por Markowitz (1952, 1959).

En su forma tradicional, el CAPM establece que la tasa de rentabilidad esperada para una firma en equilibrio $E\left(R_{i}\right)$ puede representarse por la siguiente línea de mercado de activos:

$$
E\left(R_{i}\right)=R_{f}+\left[E\left(R_{m}\right)-R_{f}\right] \cdot \beta_{i}
$$

Donde $\beta_{i}$ (beta patrimonial o simplemente el beta de la acción) representa el riesgo sistemático asociado a la empresa $i$, y se define como la covarianza entre el retorno esperado de la acción de la empresa $i$ y el retorno esperado del portafolio de mercado dividido por la varianza del retorno del portafolio de mercado. Además, $R_{f}$ corresponde a la rentabilidad del activo libre de riesgo y $R_{m}$ a la rentabilidad del portfolio de mercado. La estimación del riesgo sistemático se realiza tradicionalmente a través del modelo de mercado en excesos de retornos:

$$
R_{i t}-R_{f t}=\alpha_{i}+\beta_{i} \cdot\left(R_{m t}-R_{f t}\right)+\varepsilon_{i t}
$$

La estimación de $\alpha_{i}$ y $\beta_{i}$ se realiza regularmente a través de mínimos cuadrados ordinarios (MCO). En pocas ocasiones se utilizan mínimos 
cuadrados robustos. En algunas situaciones muy específicas se cambia el supuesto con respecto al $\beta_{i}$ y se asume que es estocástico, es decir, dependiente del tiempo $\left(\beta_{i t}\right)$. Con el propósito de hacer lo más comparable posible nuestro estudio con el de López (2016), utilizaremos MCO.

Existen varias aplicaciones del CAPM al mercado chileno en los últimos treinta años. Por ejemplo, De la Cuadra y García (1987) investigan las anomalías empíricas del CAPM y sugieren que el beta no captura todo el riesgo sistemático asociado con el nivel de endeudamiento de una firma. Sin embargo, cuando se incluye como variable explicativa —además del beta - el ratio patrimonio/total activo, los retornos anormales desaparecen y los resultados se ajustan al modelo CAPM. Por otro lado, Zúñiga $(1994,5)$ realiza una investigación exploratoria con respecto al efecto tamaño en los retornos accionarios chilenos usando datos mensuales en el período 1989-1991 y concluye que "los resultados sugieren un efecto tamaño, pero dicho efecto no es significativo en ninguna de las sub-muestras, probablemente debido al bajo número de títulos transados en Chile y a la baja presencia bursátil del promedio de las acciones usadas". Rubio (1997) trata de replicar el trabajo de Fama y French (1992) para el mercado chileno, evaluando el rol conjunto del beta de mercado, el tamaño, la razón utilidad/precio, el leverage y la razón libro/bolsa para el período 1981-1994, y usando retornos mensuales de las acciones y datos contables trimestrales y anuales. Su principal conclusión es que cuando los datos contables tienen una frecuencia trimestral, la variable más importante y que absorbe el poder explicativo de las restantes, es la razón utilidad/precio. Por el contrario, cuando la frecuencia de los datos contables es anual, la combinación más adecuada es la razón libro/bolsa y el beta de mercado.

Marshall y Walker (2000) estudian el llamado efecto del día de la semana para el período comprendido entre 1989 y 1996, usando retornos diarios, y encuentran que el día lunes los retornos de las acciones son significativamente menores a los retornos promedios diarios de la semana. Lo contrario ocurre el día viernes. Por otro lado, se detecta un efecto negativo del tamaño el día lunes y positivo el día viernes. Fuentes, Gregoire y Zurita (2006), usando rentabilidades mensuales de 60 acciones para el período 1991-2004, tratan de determinar los factores macroeconómicos que permiten explicar el rendimiento accionario en Chile. Ellos rechazan el modelo CAPM a favor de un mejor ajuste del modelo APT 
(Arbitrage Pricing Theory), modelo que explica el riesgo sistemático en base a variables macroeconómicas en un modelo factorial.

Más recientemente, Díaz e Higuera (2012) realizan un contraste empírico del CAPM empleando las metodologías de serie temporal y de eficiencia media-varianza basada en la estimación por el método generalizado de momentos (MGM). El contraste se llevó a cabo usando rentabilidades mensuales de títulos individuales para el período 1997-2007 y usando una cartera de igual ponderación como proxy para la cartera de mercado. Los resultados de todas las metodologías empleadas muestran que el CAPM explica satisfactoriamente el corte transversal de los retornos esperados en Chile.

Por último, Duarte, Ramírez y Sierra (2014) analizan los principales mercados bursátiles de la región (Argentina, Brasil, Chile, Colombia, México y Perú) mediante el análisis de la rentabilidad histórica de las empresas que cotizan en cada bolsa de valores para el período entre enero de 2002 y mayo de 2012. Los resultados obtenidos revelan que, en general, el efecto tamaño no se presenta en los principales mercados bursátiles de Latinoamérica; no obstante, se evidencia la presencia de un efecto tamaño invertido en Brasil, México, Chile y Colombia.

Cabe mencionar que para el caso de Estados Unidos, Fama y French $(1993,2015)$ muestran que, en ciertos casos, el CAPM no es capaz de explicar satisfactoriamente el comportamiento de los retornos, por lo cual proponen un modelo de tres factores (entre los cuales está el mercado) en 1993 y luego actualizan el modelo en 2015, proponiendo un modelo de cinco factores. En el caso de Chile, por limitación en el número de acciones líquidas para poder formar los portafolios de Fama y French, es difícil chequear la validez de los modelos de tres y cinco factores. Esta es una de las razones por las cuales en Chile aún se utiliza el CAPM como la base para determinar los betas de las empresas que transan en bolsa.

\section{Cálculo del CAPM para el presente estudio}

A continuación se realiza el ejercicio práctico de aplicar el CAPM a empresas chilenas, extendiendo el ejercicio realizado por López (2016) a empresas de otras industrias. Se calcula la rentabilidad exigida por el inversionista a diciembre de 2014 como pronóstico de las rentabilidades del inversionista en los siguientes cinco años (2015 a 2019). 
Se usan las empresas del IPSA por ser las más líquidas y representativas del mercado chileno. El período de estimación corresponde a los años 2010 a 2014. La alta liquidez que presentan las empresas del IPSA es un atributo importante para poder aplicar el CAPM, puesto que los precios de los activos deben actualizarse por medio de la oferta y demanda de estos; si las transacciones sufren discontinuidades, entonces el CAPM pierde capacidad explicativa. Otra virtud del IPSA es que representa a varios sectores de la economía.

\section{Estimación del riesgo sistemático}

El riesgo sistemático (o beta) es el único componente del modelo CAPM que es específico de la empresa, por lo tanto, su correcta estimación es de gran importancia para implementar adecuadamente el modelo. El problema en la práctica es que el beta de una acción en algunas ocasiones no es directamente observable y por este motivo es necesario recurrir a técnicas de estimación que permitan conocer su valor a partir de fuentes de información relevantes. La aproximación más común para su estimación es utilizar información bursátil en el contexto de un modelo de series de tiempo de los retornos de una acción que se transa en el mercado de valores, asumiendo que el riesgo sistemático es constante durante el período de estudio. Bajo estas consideraciones, el modelo CAPM implica la siguiente relación empírica, que como mencionamos anteriormente es el modelo de mercado de exceso de retornos:

$$
R_{i t}-R_{f t}=\alpha_{i}+\beta_{i} \cdot\left(R_{m t}-R_{f t}\right)+\varepsilon_{i t}
$$

Donde $R_{i t}$ es la rentabilidad total obtenida por un accionista de mantener la acción $i$ que es observada en el período $t ; R_{f t}$ es la tasa libre de riesgo observada en el período $t ; R_{m t}$ es la rentabilidad observada del portafolio de mercado en período $t ; \alpha_{i}$ es una constante para la acción $i, \beta_{i}$ es el beta de la acción $i$, y $\varepsilon_{i t}$ es un error de estimación asociado a la acción $i$.

Como mencionamos antes, los parámetros del modelo de mercado se estiman usualmente a través del método de MCO. Esta metodología, además de su simpleza, desde una perspectiva práctica es también usada por analistas del mercado de reconocido prestigio (Bloomberg, Value 
Line y Duff \& Phelps), lo cual permite grados de comparabilidad y consistencia en la forma de aplicar el método.

En cuanto a la frecuencia de los datos para estimar el beta, se han sugerido alternativas de datos diarios, semanales y mensuales. En el primer caso, sin embargo, pueden surgir comportamientos tipo GARCH (Bonilla et al. 2007, 2010) en mercados con baja liquidez como es el caso de Chile. Bloomberg usa datos semanales para un período de dos años, teniendo la ventaja de que predominan las características más actuales de la firma (especialmente en cuanto a condiciones de mercado y riesgo operacional). Por otro lado, tanto Value Line como Duff \& Phelps utilizan datos mensuales para un período de cinco años en la estimación de sus betas. En nuestro caso, se estiman los betas con retornos mensuales de la acción para cinco años, lo cual valida la comparabilidad de nuestros resultados con las estimaciones de analistas de mercado. Utilizando entonces el modelo de mercado en excesos de retornos, tenemos lo siguiente para nuestro ejercicio:

$t$ : Mes, enero de 2010 a diciembre de 2014.

$R_{i t}$ : Retorno de la acción $i$ en el mes $t$. Calculado como el logaritmo natural del precio de cierre mes $t$ dividido por el precio de cierre mensual mes $t$-1. (Bolsa de Santiago)

$R_{f t}$ : Retorno anual en el mes $t$, de un BCU/BTU-5 mensualizado, en forma continua (Banco Central, datos mercado secundario).

$R_{m t}$ : Retorno del índice IGPA en el mes $t$. Calculado como el logaritmo natural del precio de cierre mes $t$ dividido por el precio de cierre mensual mes $t$-1. (Bolsa de Santiago). Todos los retornos usados son reales, es decir, han sido ajustados por la inflación, medida también en forma continua. En el Cuadro 1 se presentan las estimaciones de riesgo sistemástico (beta) para 29 empresas del IPSA que pasaron los criterios estadísticos para ser incluidas en las estimaciones. ${ }^{2}$

\footnotetext{
${ }^{2}$ Se eliminan siete empresas por no cumplir con la propiedad de estabilidad en el beta (SQM, IL, CAP, BUPA, SMSSAM, BESALCO y SALFACORP). Hay cuatro empresas más que no presentan información en cuanto a precios de acción para el período de comparación de retornos (2015-2018). Estas empresas son CFR Pharmaceutical, que es adquirida por Abbot y deja de transar en 2015; CGE se separa en dos empresas en 2016; Endesa y Enersis se reestructuran para formar una sola empresa llamada Enel en mayo de 2018. Si contamos con precios, entonces no tenemos valores de capitalización bursátil. Finalmente, se elimina SMBCHILE porque es equivalente a considerar el Banco de Chile. El resto de las empresas tienen betas estables y estadísticamente significativos al $1 \%$ de confianza.
} 


\section{Tasa libre de riesgo}

Se utiliza como tasa libre de riesgo la TIR (Tasa Interna de Retorno) de un BCU/BTU-5 para el mes de diciembre del año 2014. Esta tasa es publicada en el Banco Central con datos del mercado secundario. Se escoge este instrumento ya que es el de menor plazo con mayor liquidez y representatividad en el mercado chileno. La tasa libre de riesgo es de 1,37\%.

\section{Premio por riesgo de mercado}

\section{Cálculos anteriores del PRM para el mercado chileno}

Fuentes y Zurita (2005) estiman el premio por riesgo de mercado (PRM) y encuentran que el valor histórico promedio es de $14,9 \%$ al usar una muestra de datos desde 1977 a 2001. Como proxy del retorno de mercado utilizan la variación del IGPA ajustado por inflación, haciendo alusión a su menor sesgo de selección. Sin embargo, los autores reconocen que el PRM estimado resulta ser inestable y no sirve como estimador del valor esperado del PRM para Chile.

Por otro lado, Lira y Sotz (2011) trabajan fundamentalmente con tres metodologías para estimar el premio por riesgo de mercado para Chile (promedio histórico, rentabilidad de mercado implícita y premio por riesgo de un mercado maduro más un premio por riesgo país). La estimación se realiza usando datos mensuales entre enero de 1993 y mayo de 2010. La primera estimación fluctuó entre 2,8\% y 6,7\%, dependiendo del índice accionario elegido y las tasas libres de riesgo empleadas. El segundo método utiliza el de dividendos descontados para deducir el retorno de mercado implícito. El PRM finalmente alcanza un rango entre 4,9\% y 7,2\% según cuál sea la tasa libre de riesgo utilizada. Por último, se realizó el cálculo adicionando el premio por riesgo país al premio por riesgo de mercado de una economía desarrollada (EE.UU.), donde el premio por riesgo de mercado fluctuó entre 3,7\% y 7,6\%. En definitiva, los autores no recomiendan el uso de alguno de los PRM obtenidos. En nuestra opinión, las series históricas de retornos de mercado para Chile son cortas (lo razonable es comenzar en 1982 dado el desarrollo del mercado accionario) y además no se cuenta con series de instrumentos libres de riesgo que no cambien sus características a través del tiempo. Es por esto que lo más aconsejable es tratar de trabajar con modelos que estimen en forma 
indirecta el PRM, o bien, el valor esperado del retorno de mercado. Por otro lado, Varas y Walker (2011) consideran para el caso de Chile la optimización de carteras tomando datos desde el primer trimestre de 1990 hasta el primer trimestre de 2008. Ellos estiman un premio por riesgo de mercado para Chile de 5,07\% para 2008. Sin embargo, es importante notar que este valor lo obtienen tomando como base para el premio por riesgo de mercado global un 4\% con respecto a bonos de largo plazo, estimado por Dimson, Marsh y Staunton (2006).

A diciembre de 2014, que es la fecha base para nuestro cálculo del CAPM, no hay más estimaciones publicadas acerca del premio por riesgo de mercado para Chile.

\section{PRM usado en nuestro caso}

Considerando la revisión de las estimaciones publicadas para el caso de Chile, nos encontramos con la limitante de que ninguno de los trabajos resuelve el problema de cuál es el PRM para Chile. En el fondo, aquel no se puede estimar como el promedio de las diferencias históricas entre el retorno de mercado y la tasa libre de riesgo, y tampoco se lo puede estimar por la vía de obtener un retorno de mercado implícito al cual se le resta la tasa libre de riesgo.

Sin embargo, Harvey (2005) plantea que para países emergentes lo recomendable es usar modelos para retornos internacionales tales como Erb, Harvey y Viskanta (1996) y Damodaran (2002). A través de cada uno de ellos se puede estimar el PRM y como estimador se puede usar el promedio simple de los tres modelos.

En nuestro caso, utilizamos la estimación del premio por riesgo de mercado realizada por la Subtel (Subsecretaría de Telecomunicaciones de Chile) en los procesos tarifarios de telefonía fija y telefonía móvil de los años 2014-2019. Este premio por riesgo de mercado se calculó utilizando la metodología de Damodaran (2013), que estima el premio por riesgo para economías emergentes sobre la base de estimaciones para economías desarrolladas. Un argumento de Damodaran es que en economías desarrolladas la medición del premio por riesgo se puede realizar con mayor confiabilidad principalmente a causa de la mayor disponibilidad de información (mayor extensión de las series de datos) y de la relativa mayor estabilidad en el crecimiento económico de estos países, 
lo cual se traduce en un menor número de cambios o quiebres estructurales. El premio por riesgo de mercado para Chile usado es entonces de 7,09\%. Cabe hacer notar que en procesos regulatorios más recientes, como es el caso del sector de transmisión eléctrica (2017) y de distribución eléctrica (2019), por ejemplo, el premio por riesgo de mercado está alrededor de un 7\%, muy cercano al usado en el presente estudio. Al respecto se puede ver Bonilla y Asociados Consultores Ltda. (2017) y Le Fort Economía y Finanzas (2019). ${ }^{3}$

\section{Resultados del CAPM}

El Cuadro 1 muestra la rentabilidad real anual exigida por el accionista de distintas empresas en varios sectores industriales a diciembre de 2014, para los cinco años siguientes. Presenta las estimaciones del beta de las 29 empresas de la muestra a través del modelo de exceso de retornos. Para el caso de la industria de AFP, ocupamos la tasa de descuento estimada para AFP Habitat, como una proxi de la industria dado que es la única AFP que transa sus acciones en la Bolsa de Comercio de Santiago, siguiendo de esta forma la misma lógica de López (2016). Sin embargo, nuestro cálculo difiere del de López (2016) por dos razones. Primero, nuestra muestra es más acotada pero al mismo tiempo más actualizada, pues considera los años 2010 al 2014 para el cálculo del CAPM. Segundo, tanto el premio por riesgo de mercado como la elección del activo libre de riesgo nuestro están basados en aplicaciones recientes del CAPM en procesos regulatorios nacionales, $y$, en consecuencia, a nuestro juicio representan de mejor manera la realidad actual del riesgo sistemático del sector empresarial nacional y de las tasas de descuento.

López (2016) señala que aplica la versión histórica del CAPM. Su estimación se basa en los siguientes supuestos. La tasa libre de riesgo es igual a $1,82 \%$ real anual y se calcula como la tasa promedio de los Bonos del Banco Central en UF a un año en el período 2006-2015. Como índice de mercado se considera el Índice General de Precios de Acciones (IGPA) de la Bolsa de Comercio de Santiago. El índice IGPA tuvo un retorno promedio de 5,86\% real anual en el período estudiado $\mathrm{y}$, por

\footnotetext{
${ }^{3}$ En ambos casos, el experto en costo de capital fue Carlos Maquieira, coautor del presente artículo.
} 
Cuadro 1. ESTIMACIÓN DE TASAS EXIGIDA (COSTO PATRIMONIAL) USANDO EL CAPM A DICIEMBRE DE 2014

\begin{tabular}{|c|c|c|c|c|c|}
\hline$N^{\circ}$ & Nombre de empresa & Beta & $\begin{array}{l}\text { Tasa libre de } \\
\text { riesgo (\%) }\end{array}$ & PRM (\%) & $\begin{array}{l}\text { Tasa exigida } \\
\text { (kp) (\%) }\end{array}$ \\
\hline 1 & AES GENER S.A. & 0,66 & 1,37 & 7,09 & 6,05 \\
\hline 2 & AFP HABITAT S.A. / SISTEMA AFP & 0,97 & 1,37 & 7,09 & 8,25 \\
\hline 3 & AGUAS ANDINAS S.A. & 0,30 & 1,37 & 7,09 & 3,50 \\
\hline 4 & ANTARCHILE & 1,16 & 1,37 & 7,09 & 9,59 \\
\hline 5 & BANCO DE CHILE & 0,92 & 1,37 & 7,09 & 7,89 \\
\hline 6 & BANCO DE CRÉDITO E INVERSIONES & 1,26 & 1,37 & 7,09 & 10,30 \\
\hline 7 & BANCO ITAÚ & 1,14 & 1,37 & 7,09 & 9,45 \\
\hline 8 & BANCO SANTANDER-CHILE & 0,98 & 1,37 & 7,09 & 8,32 \\
\hline 9 & BANCO SECURITY & 1,18 & 1,37 & 7,09 & 9,74 \\
\hline 10 & BANMÉDICA & 0,72 & 1,37 & 7,09 & 6,47 \\
\hline 11 & CENCOSUD S.A. & 1,40 & 1,37 & 7,09 & 11,30 \\
\hline 12 & COCA-COLA EMBONOR S.A. & 0,83 & 1,37 & 7,09 & 7,25 \\
\hline 13 & COLBÚN S.A. & 0,54 & 1,37 & 7,09 & 5,20 \\
\hline 14 & COMPAÑÍA CERVERÍAS UNIDAS S.A. & 0,70 & 1,37 & 7,09 & 6,33 \\
\hline 15 & EMBOTELLADORA ANDINA S.A. & 0,98 & 1,37 & 7,09 & 8,32 \\
\hline 16 & $\begin{array}{l}\text { EMPRESA NACIONAL DE } \\
\text { TELECOMUNICACIONES S.A. }\end{array}$ & 0,43 & 1,37 & 7,09 & 4,42 \\
\hline 17 & EMPRESAS CMPC S.A. & 1,31 & 1,37 & 7,09 & 10,66 \\
\hline 18 & EMPRESAS COPEC S.A. & 1,30 & 1,37 & 7,09 & 10,59 \\
\hline 19 & ENGIE ENERGÍA CHILE S.A. & 0,91 & 1,37 & 7,09 & 7,82 \\
\hline 20 & FALABELLA & 1,07 & 1,37 & 7,09 & 8,96 \\
\hline 21 & FORUS S.A. & 1,40 & 1,37 & 7,09 & 11,30 \\
\hline 22 & $\begin{array}{l}\text { INVERSIONES AGUAS } \\
\text { METROPOLITANA S.A. }\end{array}$ & 0,52 & 1,37 & 7,09 & 5,06 \\
\hline 23 & LATAM AIRLINES GROUP S.A. & 1,63 & 1,37 & 7,09 & 12,93 \\
\hline 24 & PARQUE ARAUCO S.A. & 1,00 & 1,37 & 7,09 & 8,46 \\
\hline 25 & RIPLEY CORP S.A. & 1,65 & 1,37 & 7,09 & 13,07 \\
\hline 26 & SIGDO KOPPERS S.A. & 1,58 & 1,37 & 7,09 & 12,57 \\
\hline 27 & SONDA S.A. & 1,02 & 1,37 & 7,09 & 8,60 \\
\hline 28 & VAPORES & 2,42 & 1,37 & 7,09 & 18,53 \\
\hline 29 & VIÑA CONCHA Y TORO S.A. & 0,87 & 1,37 & 7,09 & 7,54 \\
\hline
\end{tabular}

Fuente: Elaboración propia en base a información de la Bolsa de Comercio de Santiago. 
consiguiente, el premio por riesgo de mercado es igual a 4,04\% real anual (5,86\% - 1,82\%). Por último, la medida de exposición al riesgo de mercado estimada asciende a 0,86 . Con estos datos se obtiene una rentabilidad de 'equilibrio' promedio para la industria de 5,29\% real anual $\left(1,82 \%+0,86^{*} 4,04 \%\right)$. Si se desea comparar con alguna medida de rentabilidad, entonces el modelo debe predecir fuera de la muestra; en este caso, el período para la estimación es el mismo período usado para realizar la comparación de rentabilidad. En segundo lugar, el PRM calculado para Chile $(4,04 \%)$ no corresponde a un valor de equilibrio, puesto que si solo se considera el promedio histórico de Estados Unidos entre 1928 y 2010 (retorno de S\&P 500 menos la tasa de los bonos del tesoro) es igual a 6,03\%. ${ }^{4}$ Se debe tener en cuenta que Chile tiene riesgo país y, por lo tanto, el PRM debe ser mayor que el de Estados Unidos. El usado en nuestro estudio (7,09\%) es consistente con el hecho de que Chile tiene riesgo país $y$, por lo tanto, tiene un premio por sobre el del PRM de Estados Unidos.

La última columna del cuadro contiene la información clave (retorno exigido del inversionista o costo patrimonial) para la comparación que procederemos a realizar en lo que sigue. En el caso de AFP Habitat, que es nuestra proxy del sistemas de AFP (igual que López 2016), el retorno exigido es $8,25 \%$ real anual, el cual se utilizará para el sistema de AFP.

\section{Análisis comparativo con López (2016)}

López (2016) analiza la rentabilidad de la industria de las AFP utilizando el modelo CAPM y lo compara con el indicador de rentabilidad sobre el patrimonio basado en información contable (ROE). La principal conclusión de López (2016) es que la rentabilidad de las AFP ha sido 4,8 veces superior a una rentabilidad ajustada por riesgo de mercado. En efecto, en sus cálculos, al considerar el período 2006-2015, la rentabilidad sobre el patrimonio (ROE) para las AFP asciende a un $25,4 \%$ y el costo patrimonial $\left(k_{p}\right)$ calculado para la industria de AFP asciende a 5,3\%. Lo anterior lo lleva a la conclusión de que las AFP han obtenido utilidades de manera excesiva al considerar el riesgo al que dicha industria está sometida.

\footnotetext{
${ }^{4}$ Esta información es provista por Damodaran en http://people.stern.nyu.edu/adamodar/ New_Home_Page/datafile/histretSP.html [22 de septiembre 2021]. Esta información se encuentra actualmente disponible en archivos históricos.
} 
A nuestro juicio, existen tres razones por las cuales el análisis de López (2016) no debe ser considerado como prueba de exceso de rentabilidad por parte de la industria de AFP. Primero, desde la perspectiva teórica podemos decir que el CAPM es un modelo de equilibrio que nos permite calcular la tasa exigida a la que se deben descontar los flujos futuros de un proyecto, dado el riesgo particular del negocio. La tasa de descuento es una rentabilidad económica mínima exigida por los accionistas y es usada para descontar a valor presente los flujos futuros de los inversionistas. Por lo tanto, el CAPM no se puede comparar con una tasa de rentabilidad contable (ROE). ${ }^{5}$ Adicionalmente, el ROE es una medida contable ex-post, que depende no solo de los resultados del proyecto empresarial, sino también de criterios contables ${ }^{6} \mathrm{y}$, en consecuencia, tiene una mirada de pasado, y no existe razón alguna del porqué debiese ajustarse a la tasa de costo patrimonial derivada del CAPM.

La segunda razón es que la manera de calcular tanto la tasa de descuento como la rentabilidad contable sobre el patrimonio debe ser modificada en el estudio de López (2016). La tasa de costo patrimonial para la industria de AFP ya fue calculada en la sección anterior, y en nuestro caso ascendió a 8,25\%. Además, esta estimación se hace con datos fuera del período de proyección, de otra manera no tiene sentido hablar del retorno exigido esperado. En el caso de López (2016), la estimación de la tasa de descuento está dentro del período de comparación con ROE.

Para el cálculo de la rentabilidad sobre el patrimonio hemos utilizado el patrimonio bajo IFRS (International Financial Reporting Standards), método contable que nos provee de una mucho mejor aproximación al valor de mercado del patrimonio. Por lo demás, hoy en día el uso de IFRS es obligatorio para las AFP y lo que trata de hacer dicho criterio contable es reflejar el verdadero valor económico de las compañías. El uso de

\footnotetext{
${ }^{5}$ Esto lo explicaremos de forma técnica en el Anexo al final del artículo, en particular en la ecuación (1) de dicho Anexo, donde demostraremos que a partir de ROE se puede obtener una rentabilidad económica comparable con aquella rentabilidad exigida derivada del CAPM.

${ }^{6}$ En cuanto al aspecto contable, Marinovic y Valdés (2005) proponen una interesante metodología que busca reflejar de mejor forma el valor económico de los activos. Luego, para determinar la rentabilidad efectiva en un año determinado, consideran la utilidad operativa del año presente dividida por el valor económico estimado de los activos en el período anterior. A través de este método muestran una rentabilidad sobre activos de entre $35 \%$ y $68 \%$ anual, superando ampliamente la rentabilidad normal en el período 1998-2003. En todo caso, los autores reconocen la necesidad de revisar con cuidado este tema de contabilidad regulatoria.
} 
IFRS implica que el patrimonio de las AFP ahora incorpora el valor de la plusvalía producido por las reorganizaciones empresariales, lo cual se ve reflejado en la ROE contable a partir de 2015.

La tercera razón es que, en la práctica, no tiene por qué esperarse que empresa alguna tenga una tasa de descuento de equilibrio, calculada vía CAPM, que sea igual a la rentabilidad sobre el patrimonio, a menos que el valor libro del patrimonio sea siempre igual al valor del mercado del mismo. Esta es una situación improbable y, como veremos más adelante, no es el caso de las AFP. Si suscribiéramos el argumento de López (2016), muchas de las empresas nacionales de la muestra estarían obteniendo rentabilidades en exceso con respecto a lo que el modelo de equilibrio sugiere; otras estarían rentando bajo el CAPM y, en consecuencia, deberían ser vendidas para ajustarse a la línea de mercado de valores como predice el modelo para la rentabilidad de mercado. Sin embargo, la teoría financiera nos dice que esta predicción de comportamiento de ajuste al equilibrio está basada en la reacción de la oferta y la demanda a la información de la rentabilidad de mercado o bursátil, no a la rentabilidad contable.

Para explicar mejor los puntos anteriores, analicemos las empresas del IPSA a las que se les calculó la tasa de costo patrimonial $\left(k_{p}\right)$ usando el modelo CAPM y comparemos con los ROE respectivos; en el Cuadro 2 se encuentra esa comparación. Tal como es de esperar, se observa que la diferencia entre el ROE y la rentabilidad exigida $\left(k_{p}\right)$ no sigue un patrón claro. Es más, para el caso de las AFP, el ROE promedio alcanza a 14,72\% y la tasa exigida es de $8,25 \%$.

Al observar la muestra de empresas vemos que ninguna de ellas tiene una tasa de descuento igual a su rentabilidad sobre el patrimonio (columnas 6 y 7). En particular, observamos que un total de 19 empresas tienen una rentabilidad sobre el patrimonio (ROE) promedio en los últimos cuatro años mayor a la tasa de descuento de equilibrio obtenida tras la aplicación del CAPM. Además, ellas provienen de distintos sectores tales como: AES Gener, Aguas Andinas, Banco de Chile, Banmédica, Coca-Cola Embonor, Inversiones Aguas Metropolitana, Viña Concha y Toro, entre otras empresas. Estas están en la misma situación que las AFP según el criterio de López (2016). Sin embargo, ni al regulador ni a parlamentario alguno se le ocurriría proponer una intervención de dichas industrias porque están obteniendo ganancias contables por sobre las que el modelo CAPM señala que debieran hacerlo. 
Cuadro 2. CAPM ESTIMADO VERSUS ROE CONTABLE 2015-2019 (TODO EN \%)

\begin{tabular}{|c|c|c|c|c|c|c|c|c|c|}
\hline \multirow[b]{2}{*}{$\mathrm{N}^{\circ}$} & \multirow[b]{2}{*}{ Nombre Empresa } & \multicolumn{8}{|c|}{ ROE Contable } \\
\hline & & 2015 & 2016 & 2017 & 2018 & 2019 & $\begin{array}{l}\mathrm{ROE} \\
\text { prom }\end{array}$ & $\mathrm{kp}$ & ROE-kp \\
\hline 1 & AES GENER S.A. & 12,52 & 10,17 & 6,49 & 11,68 & 4,46 & 9,06 & 6,05 & 3,01 \\
\hline 2 & Sistema de AFP & 22,34 & 12,68 & 12,09 & 10,20 & 16,28 & 14,72 & 8,25 & 6,47 \\
\hline 3 & AGUAS ANDINAS S.A. & 18,54 & 21,56 & 19,91 & 19,20 & 20,12 & 19,86 & 3,50 & 16,37 \\
\hline 4 & ANTARCHILE & 3,32 & 2,92 & 3,36 & 6,62 & 1,18 & 3,48 & 9,59 & $-6,11$ \\
\hline 5 & BANCO DE CHILE & 21,11 & 19,38 & 19,54 & 18,74 & 17,50 & 19,25 & 7,89 & 11,36 \\
\hline 6 & $\begin{array}{l}\text { BANCO DE CRÉDITO E } \\
\text { INVERSIONES }\end{array}$ & 17,59 & 8,01 & 14,44 & 14,19 & 11,35 & 13,12 & 10,30 & 2,81 \\
\hline 7 & BANCO ITAÚ & 5,65 & 0,31 & 1,65 & 4,95 & 3,49 & 3,21 & 9,45 & $-6,24$ \\
\hline 8 & BANCO SANTANDER-CHILE & 16,26 & 16,43 & 19,09 & 18,63 & 16,34 & 17,35 & 8,32 & 9,03 \\
\hline 9 & BANCO SECURITY & 11,72 & 12,54 & 12,08 & 10,90 & 10,57 & 11,56 & 9,74 & 1,83 \\
\hline 10 & BANMÉDICA & 20,64 & 17,26 & 17,03 & 11,16 & 13,58 & 15,94 & 6,47 & 9,46 \\
\hline 11 & CENCOSUD S.A. & 4,97 & 9,39 & 10,55 & 4,67 & 2,62 & 6,44 & 11,30 & $-4,86$ \\
\hline 12 & COCA-COLA EMBONOR S.A. & 8,54 & 11,58 & 11,82 & 12,21 & 12,27 & 11,29 & 7,25 & 4,03 \\
\hline 13 & COLBÚN S.A. & 6,82 & 5,00 & 6,43 & 6,71 & 5,57 & 6,11 & 5,20 & 0,91 \\
\hline 14 & $\begin{array}{l}\text { COMPAÑÍA CERVECERÍAS } \\
\text { UNIDAS S.A. }\end{array}$ & 10,07 & 9,59 & 10,57 & 24,47 & 9,14 & 12,77 & 6,33 & 6,44 \\
\hline 15 & $\begin{array}{l}\text { EMBOTELLADORA ANDINA } \\
\text { S.A. }\end{array}$ & 9,16 & 10,22 & 13,70 & 11,62 & 19,62 & 12,86 & 8,32 & 4,54 \\
\hline 16 & $\begin{array}{l}\text { EMPRESA NACIONAL DE } \\
\text { TELECOMUNICACIONES S.A. }\end{array}$ & $-0,11$ & 3,44 & 3,37 & $-1,87$ & 11,00 & 3,17 & 4,42 & $-1,25$ \\
\hline 17 & EMPRESAS CMPC S.A. & $-0,10$ & $-0,20$ & 1,17 & 6,85 & 1,08 & 1,76 & 10,66 & $-8,90$ \\
\hline 18 & EMPRESAS COPEC S.A. & 5,83 & 5,11 & 5,48 & 10,82 & 1,63 & 5,77 & 10,59 & $-4,81$ \\
\hline 19 & ENGIE ENERGÍA CHILE S.A. & 5,91 & 12,69 & 4,52 & 5,48 & 5,48 & 6,82 & 7,82 & $-1,01$ \\
\hline 20 & FALABELLA & 11,98 & 12,17 & 9,89 & 8,84 & 4,81 & 9,54 & 8,96 & 0,58 \\
\hline 21 & FORUS S.A. & 20,04 & 14,59 & 13,88 & 12,26 & 10,33 & 14,22 & 11,30 & 2,92 \\
\hline 22 & $\begin{array}{l}\text { INVERSIONES AGUAS } \\
\text { METROPOLITANA S.A. }\end{array}$ & 6,46 & 7,58 & 7,02 & 6,81 & 7,11 & 6,99 & 5,06 & 1,94 \\
\hline 23 & LATAM AIRLINES GROUP S.A. & $-5,44$ & 2,14 & 3,34 & 8,01 & 5,85 & 2,78 & 12,93 & $-10,15$ \\
\hline 24 & PARQUE ARAUCO S.A. & 7,38 & 8,68 & 9,69 & 12,25 & 8,45 & 9,29 & 8,46 & 0,83 \\
\hline 25 & RIPLEY CORP S.A. & 4,63 & 8,91 & 9,93 & 7,21 & 9,86 & 8,11 & 13,07 & $-4,96$ \\
\hline 26 & SIGDO KOPPERS S.A. & 9,42 & 4,91 & 4,46 & 5,39 & 6,36 & 6,11 & 12,57 & $-6,46$ \\
\hline 27 & SONDA S.A. & 8,16 & 5,45 & 12,55 & 2,13 & 4,52 & 6,56 & 8,60 & $-2,04$ \\
\hline 28 & VAPORES & $-0,81$ & $-1,09$ & $-8,37$ & 0,97 & 6,23 & $-0,61$ & 18,53 & $-19,14$ \\
\hline 29 & VIÑA CONCHA Y TORO S.A. & 10,76 & 10,14 & 9,38 & 8,71 & 8,95 & 9,59 & 7,54 & 2,05 \\
\hline
\end{tabular}

Fuente: Elaboración propia en base a información de la Comisión del Mercado Financiero. 


\section{Discusión}

Uno de los problemas más relevantes con los que se encuentran los policymakers dice relación con la falta de información sobre los reales efectos de las políticas públicas que ellos mismos impulsan. En particular, la falta de estudios ex-ante sobre la real necesidad de realizar cambios regulatorios o impulsar nuevas regulaciones o programas públicos en los mercados para mejorar la eficiencia y competitividad de ellos, sin duda son un desafío. Por otro lado, la falta de estudios ex-post sobre los impactos y efectos que dichos cambios regulatorios o políticas públicas causan en los participantes del mercado, dejan abierta la puerta a decisiones basadas no en evidencia, sino más bien en la ignorancia, ideología, wishful thinking o alternativamente en un potencial de figuración política y popularidad rápida, en particular en períodos cercanos a las elecciones. Es por eso que un análisis más profundo, basado en evidencia y en los principios de la ciencia económica, nunca está de más para clarificar ideas que circulan en los medios y que requieren una mirada más racional a la luz de los hechos.

Es en ese contexto que en el presente estudio hemos querido profundizar la discusión respecto del nivel de rentabilidad que alcanzan las AFP. Para lograrlo hemos extendido y mejorado la metodología aplicada por López (2016) al sector de las AFP, la cual hemos empleado en 29 empresas del IPSA de distintos sectores económicos para concluir que el cálculo realizado por López (2016) sobre excesos de rentabilidad del sector, simplemente no es pertinente de realizar. Primero, como argumentamos extensamente en el artículo, porque la mirada contable histórica nada tiene que ver con el modelo CAPM de equilibrio, cuya mirada es económica y enfocada en el futuro. Segundo, porque en el proceso de replicación de la metodología de López (2016) en otros sectores económicos, el diferencial entre la rentabilidad contable y el retorno exigido por los accionistas es mucho mayor, y a nadie se le ocurre pedir la intervención de dichos sectores. Por último, es importante mencionar que el concepto de retornos sobrenormales en finanzas es un concepto de mercado, en el sentido de que activos con retornos esperados mayores de lo que su nivel de riesgo predice — según el modelo de mercado-, verá aumentada su demanda y ajustada su rentabilidad esperada, y esto nada tiene que ver con el gradiente contable en dicho análisis de rentabilidad en exceso. 
En esta discusión hemos querido desmitificar ideas sobre excesos de rentabilidad en la industria de las AFP, y contextualizar dichas rentabilidades comparativamente con otras industrias que incluyen, no solo otras empresas del ámbito financiero como los bancos o aseguradoras de salud, sino también empresas que son monopolios naturales regulados y cuya rentabilidad económica está definida por ley.

\section{Conclusiones}

Hemos replicado el ejercicio empírico de López (2016), pero esta vez no solo para la industria de las AFP, sino también para empresas de variadas industrias, tanto en mercados competitivos como en mercados regulados.

La principal conclusión a la que llegamos es que el costo de capital calculado vía el método del CAPM no tiene por qué ser parecido a la rentabilidad contable sobre el patrimonio. Los cálculos realizados para las empresas que componen el IPSA muestran claramente la gran distancia entre el ROE y la tasa de rentabilidad exigida (costo patrimonial).

En particular, recalculamos el costo patrimonial para la industria de las AFP acorde a datos actualizados y usados en procesos tarifarios recientes. De estos procesos obtuvimos los datos para el activo libre de riesgo y el premio por riesgo de mercado. Nuestro cálculo para el costo de capital de las AFP asciende a 8,25\%. Además, calculamos el índice de rentabilidad sobre el patrimonio (ROE) de la industria de AFP, considerando los últimos cinco años desde los estados financieros bajo IFRS - metodología actualmente obligatoria para las AFP — para llegar a un índice de $14,72 \%$. Lo mismo hicimos para otras 28 empresas de la muestra y la principal conclusión a la que llegamos es que el diferencial ROE/ CAPM puede ser positivo o negativo, pero en ningún caso está disparado para las AFP respecto de cualquier otra industria, incluso al compararlo con empresas pertenecientes a industrias reguladas.

Los resultados anteriores son consistentes con la idea de que, en principio, la comparación entre costo patrimonial vía CAPM y la rentabilidad contable sobre el patrimonio no es la forma correcta de calcular retornos o ganancias en exceso. El CAPM tiene que ver con la rentabilidad mínima exigida a los flujos futuros de un proyecto empresarial, dado el riesgo que dicha compañía enfrenta (son las 'peras'), mientras que la ren- 
tabilidad contable tiene que ver con la historia pasada de una compañía y con los criterios contables aplicados en la confección de sus estados financieros (son las 'manzanas').

Alguien podría pensar que en el largo plazo, en un estado estable de equilibrio como decimos los economistas, y con una contabilidad objetiva y perfecta, donde nada queda al criterio subjetivo del contador y donde además dicha contabilidad refleja perfectamente la situación económica del presente y del futuro de la firma, es decir, en una contabilidad económica que mira hacia adelante y también considera la historia de la empresa, efectivamente el CAPM debiera asimilarse a la rentabilidad contable. En este caso tendríamos que el valor económico del patrimonio es igual al valor contable del mismo. El problema es que dicha contabilidad objetiva y futurista no existe. Además, como decía el gran John Maynard Keynes (1936), en el largo plazo estamos todos muertos, lo que significa que el famoso 'estado estable' es un constructo intelectual que sirve fundamentalmente para escribir papers, no para aportar recomendaciones de políticas públicas, pues en la práctica siempre vamos hacia allá pero nunca llegamos.

Por último, en el Anexo técnico hemos desarrollado la relación del ROE con el retorno esperado de una acción (R). Esta relación pasa por ajustar ROE por la relación B/M (book-to-market ratio) y por los movimientos que experimenten las diferencias entre el patrimonio económico y el contable. Al respecto observamos que cuando se aplica el método correcto de comparación, se muestra que la diferencia entre el retorno económico y el retorno exigido (costo patrimonial) es bastante más atenuado. Además, para el caso de las AFP se demuestra que la rentabilidad económica está por debajo de la tasa exigida (costo patrimonial) al utilizar correctamente la relación entre ROE y R.

\section{Bibliografía}

Bonilla, C., Hinich M. y Romero, R. 2007. GARCH Inadequacy for Modelling Exchange Rates: Empirical Evidence from Latin America. Applied Economics 39, 2529-2533.

Bonilla, C., Romero, R. y Borquez, R. 2010. Intraday Patterns in Exchange Rate of Return of the Chilean Peso: New Evidence for the Day-of-the-Week Effect. Macroeconomic Dynamics 14(S1), 42-58.

Bonilla y Asociados Consultores Ltda. 2017. Metodología de cálculo para la tasa de descuento de una empresa eficiente de transmisión eléctrica. Comisión Nacional de Energía, Chile. 
Damodaran, A. 2002. Investment Valuation. New York: John Wiley \& Sons.

Damodaran, A. 2013. Equity Risk Premiums (ERP): Determinants, Estimation and Implications. The 2013 Edition. SSRN Working Paper 2238064.

De la Cuadra, R. y García, V. 1987. Modelo de valuación de activos de capital y riesgo financiero. Cuadernos de Economía 24(73), 359-374.

Díaz, C. y Higuera, F. 2012. Contraste empírico del CAPM en el mercado accionario chileno. Ingeniare. Revista Chilena de Ingeniería 20(2), 255-266.

Dimson, E., Marsh, P. y Staunton, M. 2006. The Worldwide Equity Premium: A Smaller Puzzle. SSRN Working Paper 891620.

Duarte, J., Ramírez, Z. y Sierra, K. 2014. Estudio del efecto tamaño en los principales mercados bursátiles de Latinoamérica. Revista Internacional de Administración y Finanzas 7(5), 41-50.

Easton, P., Harris, T. y Ohlson, J. 1992. Aggregate Accounting Earnings Can Explain most of Security Returns: The Case of Long Event Windows. Journal of Accounting and Economics 15, 119-142.

Erb, C.B., Harvey, C.R. y Viskanta, T.E. 1996. Expected Returns and Volatility in 135 Countries. The Journal of Portfolio Management 22(3), 46-58.

Fama, E.F. y French, K.R. 1992. The Cross-Section of Expected Stock Returns. Journal of Finance 47(2), 427-465.

Fama, E.F. y French, K.R. 1993. Common Risk Factors in the Returns on Stocks and Bonds. Journal of Financial Economics 33(1), 3-56.

Fama, E.F. y French, K.R. 2015. A Five-Factor Asset Pricing Model. Journal of Financial Economics 116(1), 1-22.

Fuentes, R., Gregoire, J. y Zurita, S. 2006. Factores macroeconómicos en rendimientos accionarios chilenos. El Trimestre Económico 73(289), 125-138.

Fuentes, J.R. y Zurita, S. 2005. The Equity Risk Premium in Emerging Markets: The Case of Chile. Monetaria 28(3), 223-254.

Harvey, C.R. 2005. 12 Ways to Calculate the International Cost of Capital. Fuqua School of Business, Duke University. Manuscrito.

Keynes, J.M. 1936. The General Theory of Employment, Interest and Money. London: Macmillan.

Larocque, S. y Lyle, M. 2017. Implied Cost of Equity Capital Estimates as Predictors of Accounting Returns and Stock Returns. Journal of Financial Reporting 2(1), 69-93.

Le Fort Economía y Finanzas 2019. Metodología de cálculo para la tasa de actualización de una empresa eficiente de distribución eléctrica. Comisión Nacional de Energía, Chile.

Lintner, J. 1965. The Valuation of Risk Assets and the Selection of Risky Investments in Stock Portfolios and Capital Budgets. Review of Economics and Statistics 47(1), 13-37.

Lira, F. y Sotz, C. 2011. Estimación del premio por riesgo en Chile. Central Bank of Chile Working Papers 617.

López, F. 2016. Industria de AFP chilena: ¿cuánto gana y cuanto debería ganar? Revista de Análisis Económico 31(2), 101-114.

Maquieira y Asociados 2017. Estimación del costo de capital para empresas de distribución de gas en red. Informe elaborado para Panel de Expertos, Chile.

Marcel, M. et al. 2008. El derecho a la vida digna en la vejez. Hacia un contrato social con la previsión en Chile. Santiago: Consejo Asesor Presidencial para la Reforma Previsional. Disponible en: https://www.previsionsocial.gob.cl/sps/download/ estudios-previsionales/comisionpensiones/documentos-interes-general/ 
informe-consejo-asesor-presidencial-reforma-del-sistema-previsional-comisionmarcel-2006.pdf [19 de septiembre 2021].

Marinovic, I. y Valdés, S. 2005. Contabilidad regulatoria: las AFP chilenas, 1993-2003. Documento de trabajo 279. Instituto de Economía PUC.

Markowitz, H. 1952. Portfolio Selection. Journal of Finance 7(1), 77-91.

Markowitz, H. 1959. Portfolio Selection: Efficient Diversification of Investments. Cowles Foundation Monograph. New York: John Wiley \& Sons, Inc. Monograph No. 16.

Marshall, P. y Walker, E. 2000. Day-of-the Week and Size Effects in Emerging Markets: Evidence from Chile. Revista de Análisis Económico 15(2), 89-108.

Penman, S. 2016. Valuation: Accounting for Risk and the Expected Return. Abacus 52(1), 106-130.

Rubio, F. 1997. Corte transversal de los retornos en el mercado accionario chileno, entre enero de 1981 y abril de 1994. Tesis (Mg) en Administración, mención Finanzas. Universidad de Chile.

Sharpe, W.F. 1964. Capital Asset Prices: A Theory of Market Equilibrium under Conditions of Risk. Journal of Finance 19(3), 425-442.

Varas, F. y Walker, E. 2011. Optimal Close-to-Home Biases in Asset Allocation. Journal of Business Research 64 (3), 328-337.

Zúñiga, S. 1994. El CAPM en Chile: evidencia de regularidades empíricas. Revista Escuela de Administración de Negocios 23, 5-13.

\section{Anexo técnico}

\section{Comparación del retorno exigido, obtenido a través de CAPM/ROE en distintas industrias}

Es posible establecer una relación entre el retorno contable de una acción (ROE) y su retorno económico (R). Para ello basta con recurrir a los fundamentos del modelo de valoración desarrollado por Penman (2016).

El retorno económico de una acción equivale a:

$$
R_{t+1}=\frac{M_{t+1}+D_{t+1}}{M_{t}}-1
$$

Donde $M_{t+1}$ es el valor de mercado del patrimonio (market capitalization) en $t+1 ; D_{t+1}$ corresponde al pago total de dividendos en $t+1$. Lo anterior es equivalente a decir que el retorno de una acción se descompone en dos retornos: retorno por dividendos $\left(\frac{D_{t+1}}{M_{t}}\right)$ y retorno por ganancias de capital $\left(\frac{M_{t+1}-M_{t}}{M_{t}}\right)$.

Por su parte, $R O E_{t+1}$ se puede expresar de la siguiente forma: 


$$
R O E_{t+1}=\frac{B_{t+1}+D_{t+1}}{B_{t}}-1
$$

Donde $B_{t+1}$ es el valor libro del patrimonio en $t+1$.

Considerando la siguiente identidad:

$$
B_{t+1}=B_{t}+\text { Earnings }_{t+t}-D_{t+1}
$$

Entonces, resolviendo para $D_{t+1}$ en la ecuación anterior, luego sustituyendo el resultado en la ecuación de retorno económico de la acción $\left(R_{t+1}\right)$, se obtiene que:

$$
R_{t+1}=\frac{\text { Earnings }_{t+1}}{M_{t}}+\frac{M_{t+1}-B_{t+1}-\left(M_{t}-B_{t}\right)}{M_{t}}
$$

La ecuación anterior ya había sido claramente reconocida para establecer la relación entre retornos económicos realizados $\left(R_{t+1}\right)$ y retornos contables realizados (Easton, Harris y Ohlson 1992).

A partir de la ecuación anterior se puede establecer una relación entre el retorno contable del patrimonio $\left(R O E_{t+1}\right)$ y el retorno económico del mismo $\left(R_{t+1}\right)$ tal como sigue:

$$
R_{t+1}=R O E_{t+1} \frac{B_{t}}{M_{t}}+\frac{\left(M_{t+1}-B_{t+1}\right)-\left(M_{t}-B_{t}\right)}{M_{t}}
$$

Por lo tanto, el retorno económico de la acción del próximo período $\left(R_{t+1}\right)$ es la rentabilidad del patrimonio contable del próximo período $\left(R O E_{t+1}\right)$ ajustado por $\frac{B_{t}}{M_{t}}$ (book-to-market año anterior), más el cambio esperado del premio relacionado a la diferencia entre el valor de mercado del patrimonio y el valor libro del mismo $\left[\frac{M_{t+1}-B_{t+1}-\left(M_{t}-B_{t}\right)}{M_{t}}\right]$. No debemos olvidar que (1) es una tautología; no requiere ningún supuesto especial para obtener esa igualdad $y$, por lo tanto, a partir de conocer $R O E_{t+1}, B_{t}, B_{t+1}, M_{t}$ y $M_{t+1}$ podemos obtener el retorno económico de la acción. Este retorno económico es comparable a la medida de retorno económico exigido. Finalmente, se deduce de la ecuación (1) que para que el retorno económico $\left(R_{t+1}\right)$ sea igual al $R O E_{t+1}$ se hace necesario que el valor libro y de mercado del patrimonio sean iguales, en cada período. Esto dependerá de cada caso y lo más probable es que el valor del pa- 
trimonio contable difiera del valor económico, por lo cual es muy poco probable que ambos retornos sean iguales.

Larocque y Lyle (2017) plantean el siguiente modelo para establecer la relación entre las tres variables:

$$
\operatorname{roe}_{t+i}=\propto_{0}+\propto_{1} \hat{\mu}+\propto_{2} b m_{t}+\varepsilon_{t+i}
$$

Conforme a la ecuación anterior es imposible explicar roe solo a través del retorno esperado de la acción $(\hat{\mu})$ sino que es necesario controlar por bm (B/M). Ellos muestran claramente que existe una relación estadísticamente significativa entre roe y $\mathrm{bm}$, así como con el retorno esperado de la acción $(\hat{\mu})$. Mayores niveles de $\mathrm{B} / \mathrm{M}$ y expectativas más altas de ROE llevan a mayores retornos económicos esperados. Como las estimaciones de López (2016) no están hechas para betas estocásticos, entonces lo razonable es usar la ecuación (1) para poder hacer comparaciones.

Ahora calcularemos la rentabilidad económica (R) que es comparable con la rentabilidad exigida (costo patrimonial). Para esto usaremos la ecuación 1. En el Cuadro 3 se pueden apreciar los resultados obtenidos. Hemos calculado la rentabilidad económica promedio para el período 2015-2018 (R1) y luego el promedio para 2015-2019 (R2). Esto, considerando las circunstancias especiales que se vivieron en el país entre octubre y diciembre de 2019, producto del llamado estallido social y del vandalismo callejero - de hecho, gran parte de las empresas tuvieron un ROE negativo en 2019.

En el Cuadro 3 se puede apreciar claramente que la comparación entre ROE y retorno exigido (Cuadro 2) lleva a conclusiones distintas cuando se compara retorno económico (R) con retorno exigido. Solo si comparamos ganancias y pérdidas, para 11 de los 29 casos mostrados en ambos cuadros, vemos que bajo un criterio hay ganancia y bajo el otro, pérdida o viceversa. Esto, haciendo un análisis básico, puesto que si se analizan las diferencias nos encontramos con grandes variaciones. Por ejemplo, bajo el criterio de López (2016), con ROE se estima un exceso de rentabilidad de 22,85\% para el sistema de AFP, mientras que bajo el criterio de comparación de retornos económicos con base en el ROE la diferencia es $-3,05 \%$, pues el sistema tuvo una rentabilidad eco- 
Cuadro 3. CAPM ESTIMADO VERSUS RENTABILIDAD ECONÓMICA (R) OBTENIDA A PARTIR DE ROE, 2015-2019

\begin{tabular}{|c|c|c|c|c|c|c|c|c|c|c|c|}
\hline$N^{\circ}$ & Nombre empresa & 2015 & 2016 & 2017 & 2018 & 2019 & $\mathrm{R} 1$ & R2 & Kp & R-kp & $\mathrm{R} 2-\mathrm{kp}$ \\
\hline 1 & AES GENER S.A. & $-5,45$ & $-20,33$ & $-7,04$ & $-7,46$ & $-10,11$ & $-10,07$ & $-10,08$ & 6,05 & $-16,12$ & $-16,13$ \\
\hline 2 & AFP HABITAT S.A. & $-7,74$ & 2,75 & 23,52 & 1,57 & $-19,01$ & 5,02 & 0,22 & 8,25 & $-3,22$ & $-8,03$ \\
\hline 3 & AGUAS ANDINAS S.A. & 6,06 & $-0,47$ & 21,02 & $-1,84$ & $-11,32$ & 6,19 & 2,69 & 3,50 & 2,70 & $-0,81$ \\
\hline 4 & ANTARCHILE & $-18,91$ & 14,22 & 80,77 & $-26,50$ & $-29,59$ & 12,39 & 4,00 & 9,59 & 2,80 & $-5,60$ \\
\hline 5 & BANCO DE CHILE & 6,53 & 13,33 & 30,65 & 4,58 & $-17,21$ & 13,77 & 7,58 & 7,89 & 5,88 & $-0,32$ \\
\hline 6 & $\begin{array}{l}\text { BANCO DE CRÉDITO E } \\
\text { INVERSIONES }\end{array}$ & $-6,65$ & 27,14 & 30,02 & 7,12 & $-20,64$ & 14,41 & 7,40 & 10,30 & 4,10 & $-2,91$ \\
\hline 7 & BANCO ITAÚ & 19,56 & $-85,07$ & 2,10 & 16,67 & $-24,84$ & $-11,68$ & $-14,32$ & 9,45 & $-21,14$ & $-23,77$ \\
\hline 8 & BANCO SANTANDER-CHILE & 7,81 & 19,92 & 32,46 & 10,13 & $-14,37$ & 17,58 & 11,19 & 8,32 & 9,26 & 2,87 \\
\hline 9 & BANCO SECURITY & $-8,01$ & 23,69 & 33,29 & 8,60 & $-28,53$ & 14,39 & 5,81 & 9,74 & 4,65 & $-3,93$ \\
\hline 10 & BANMÉDICA & $-15,80$ & 26,51 & 66,66 & $-8,61$ & 3,08 & 17,19 & 14,37 & 6,47 & 10,72 & 7,89 \\
\hline 11 & CENCOSUD S.A. & 5,54 & 35,88 & 6,34 & $-31,49$ & $-37,56$ & 4,07 & $-4,26$ & 11,30 & $-7,23$ & $-15,55$ \\
\hline 12 & COCA-COLA EMBONOR S.A. & $-11,09$ & $-15,50$ & 19,03 & $-4,55$ & $-12,62$ & $-3,03$ & $-4,94$ & 7,25 & $-10,28$ & $-12,20$ \\
\hline 13 & COLBÚN S.A. & 36,76 & $-9,14$ & 34,07 & $-0,56$ & $-16,08$ & 15,28 & 9,01 & 5,20 & 10,09 & 3,81 \\
\hline 14 & $\begin{array}{l}\text { COMPAÑÍA CERVECERÍAS } \\
\text { UNIDAS S.A. }\end{array}$ & 34,43 & 19,83 & 28,42 & $-17,72$ & $-14,44$ & 16,24 & 10,10 & 6,33 & 9,91 & 3,77 \\
\hline 15 & EMBOTELLADORA ANDINA S.A. & 12,62 & 31,12 & 26,87 & $-5,72$ & $-7,08$ & 16,22 & 11,56 & 8,32 & 7,90 & 3,24 \\
\hline 16 & $\begin{array}{l}\text { EMPRESA NACIONAL DE } \\
\text { TELECOMUNICACIONES S.A. }\end{array}$ & 2,40 & 22,60 & $-0,47$ & $-29,67$ & $-6,29$ & $-1,28$ & $-2,28$ & 4,42 & $-5,70$ & $-6,70$ \\
\hline 17 & EMPRESAS CMPC S.A. & $-14,24$ & $-1,65$ & 65,16 & $-2,12$ & $-22,08$ & 11,79 & 5,02 & 10,66 & 1,13 & $-5,64$ \\
\hline 18 & EMPRESAS COPEC S.A. & $-15,48$ & 8,85 & 58,23 & $-17,37$ & $-23,79$ & 8,56 & 2,09 & 10,59 & $-2,03$ & $-8,50$ \\
\hline 19 & ENGIE ENERGÍA CHILE S.A. & $-5,00$ & 21,13 & 36,49 & $-14,04$ & $-11,51$ & 9,64 & 5,41 & 7,82 & 1,82 & $-2,41$ \\
\hline 20 & FALABELLA & 6,48 & 17,78 & 16,18 & $-16,67$ & $-36,60$ & 5,94 & $-2,57$ & 8,96 & $-3,01$ & $-11,52$ \\
\hline 21 & FORUS S.A. & $-29,18$ & 28,16 & 23,19 & $-31,01$ & $-40,65$ & $-2,21$ & $-9,90$ & 11,30 & $-13,51$ & $-21,20$ \\
\hline 22 & $\begin{array}{l}\text { INVERSIONES AGUAS } \\
\text { METROPOLITANA S.A. }\end{array}$ & 11,96 & 2,21 & 26,80 & $-7,24$ & $-12,01$ & 8,43 & 4,34 & 5,06 & 3,38 & $-0,71$ \\
\hline 23 & LATAM AIRLINES GROUP S.A. & $-34,21$ & 34,01 & 60,81 & $-12,72$ & 11,59 & 11,97 & 11,90 & 12,93 & $-0,95$ & $-1,03$ \\
\hline 24 & PARQUE ARAUCO S.A. & 2,36 & 41,01 & 27,38 & $-20,27$ & 18,89 & 12,62 & 13,87 & 8,46 & 4,16 & 5,41 \\
\hline 25 & RIPLEY CORP S.A. & 3,87 & 41,33 & 71,51 & $-12,08$ & $-35,87$ & 26,16 & 13,75 & 13,07 & 13,09 & 0,68 \\
\hline 26 & SIGDO KOPPERS S.A. & $-14,77$ & 5,82 & 61,28 & $-21,68$ & $-8,14$ & 7,66 & 4,50 & 12,57 & $-4,91$ & $-8,07$ \\
\hline 27 & SONDA S.A. & $-8,96$ & $-8,54$ & 9,51 & $-11,99$ & $-41,82$ & $-4,99$ & $-12,36$ & 8,60 & $-13,60$ & $-20,96$ \\
\hline 28 & VAPORES & $-78,20$ & 56,85 & 115,04 & $-52,49$ & 26,88 & 10,30 & 13,62 & 18,53 & $-8,22$ & $-4,91$ \\
\hline \multirow[t]{3}{*}{29} & VIÑA CONCHA Y TORO S.A. & $-8,07$ & $-2,30$ & 9,40 & 18,33 & 6,28 & 4,34 & 4,73 & 7,54 & $-3,20$ & $-2,81$ \\
\hline & Sistemas de AFP* & 6,16 & 4,40 & 4,71 & 3,82 & 6,91 & 4,77 & 5,20 & 8,25 & $-3,47$ & $-3,05$ \\
\hline & Promedio & & & & & & 8,17 & 3,53 & 8,91 & $-0,74$ & $-5,38$ \\
\hline
\end{tabular}

* Sin considerar premios o descuentos en variaciones del valor económico y contable del patrimonio. R1 es promedio (2015-2018); R2 promedio (2015-2019).

Fuente: Elaboración propia. 
nómica promedio en el período de 5,20\%, ${ }^{7}$ mientras que la rentabilidad exigida es $8,03 \%$. En cuanto al resultado general para la muestra de empresas, en el Cuadro 3 se obtiene una rentabilidad promedio de $8,17 \%$ (R1) y el promedio de la rentabilidad exigida es $8,91 \%$, resultando una pérdida de $0,74 \%$. A través de un test de diferencias de media hemos comprobado que no existe una ganancia anormal al 1\% de confianza. Es de esperar que en un mercado eficiente, donde el CAPM explica apropiadamente los retornos, los retornos económicos realizados (R1) no debiesen diferir de los retornos exigidos, en la medida en que la muestra de datos sea representativa. En general, esta muestra es adecuada para realizar comparaciones; así también lo es el período (20152018) puesto que de no serlo entonces deberíamos observar diferencias estadísticamente significativas entre el retorno promedio económico de toda la muestra y el retorno promedio exigido de la misma. EP

\footnotetext{
${ }^{7}$ Para evitar una potencial subvaloración de la rentabilidad económica, se ha supuesto que solo se ajusta el ROE por la relación entre el valor libro y el valor de mercado del patrimonio (2015-2018) mostrado por AFP Habitat (valor entre 0,28 y 0,39), y se supone que el segundo término de la ecuación (1) es cero, a pesar de que para AFP Habitat el valor promedio es de $-3,40 \%$.
} 